\title{
Estrategia Internacional para la seguridad humana en los desastres naturales
}

\section{International Strategy on Human Security in Times of Natural Disasters}

\author{
Helena Torroja Mateu ${ }^{1}$ \\ Universidad de Barcelona (España)
}

Recibido: 19-02-16

Aprobado: 05-04-16

\section{Resumen}

Este artículo presenta los rasgos principales de la estrategia internacional universal de seguridad humana en casos de desastres naturales liderada por la ONU. La estrategia se basa en dos grandes funciones: la prevención y preparación ante desastres naturales y la movilización y coordinación de la asistencia humanitaria a las víctimas, incluida la de los propios órganos operacionales de la ONU. Del desarrollo de ambas funciones se encargan diversos órganos operacionales (UNISDIR, PNUD, OCHA...). Con respecto a ambas finalidades se han adoptado directrices políticas y normativas por la Asamblea General. Destaca en este sentido el interés actual de la CDI, quien trabaja en un proyecto de artículos sobre la protección de personas en casos de desastres.

Palabras-clave: Desastres Naturales, Seguridad Humana, Naciones Unidas, Protección y Asistencia Humanitaria, Prevención y Preparación Ante Desastres.

\footnotetext{
${ }^{1}$ (htorroja@ub.edu) Profesora agregada de Derecho internacional público de la Universidad de Barcelona (Titular ANECA); Directora Académica del Centro de Estudios Internacionales (UBMAEC-LaCaixa); Consultora del ACNUDH. Investigadora visitante en el Washington College of Law de la American University (2016). Entre sus publicaciones recientes se encuentran: (con Pelegrin, A.) (Ed.), China hoy: claves para entender su liderazgo en el tablero internacional (2014) y (con otros autores) El arma de moda: impacto del uso de los drones en las relaciones internacionales y el derecho internacional contemporáneo (2014).
} 


\begin{abstract}
This article analyses the principal features of the United Nation's international strategy on human security in times of natural disasters. This strategy focuses on two main principles: prevention and preparedness for natural disasters, as well as the mobilization and coordination of humanitarian assistance for the victims, including assistance provided by UN agencies. Several agencies are in charge of these functions (UNISDIR, UNDP, OCHA...). The General Assembly has adopted political and normative guidelines to uphold both of these principles. This sheds light on the ILC's current work, a project to draft articles on the protection of persons in the event of disasters.
\end{abstract}

Key-words: Natural Disasters, Human Security, United Nations, Protection and Humanitarian Assistance, Prevention and Preparedness For Disasters.

\title{
1. Introducción
}

A finales de la década de los años sesenta del siglo XX, la Organización de las Naciones Unidas (ONU en adelante) hizo suyo el interés común de los Estados de fomentar la cooperación ante las situaciones de desastres naturales. Desde entonces hasta nuestros días la competencia material atribuida por los Estados a la Organización en este sector, se ha ido ampliando y consolidando, concretándose en diversas formas de cooperación internacional dirigidas a armonizar y coordinar las políticas y comportamientos estatales así como a movilizar y coordinar la prestación de asistencia humanitaria, principalmente. Es posible por tanto afirmar que hoy existe en la ONU, la organización internacional universal de la que forman parte la práctica totalidad de los Estados de la sociedad internacional contemporánea, una estrategia internacional para prevenir y afrontar las consecuencias de los desastres naturales. Esta estrategia internacional, en la medida en que tiene en su centro de mira la protección y asistencia del ser humano, puede muy bien denominarse como Estrategia Internacional para la Seguridad Humana en los Desastres Naturales, tal como indica el título de este artículo, que acertadamente -en la elección de la denominación- ha propuesto el coordinador de este monográfico. Las presentes páginas tienen por objeto identificar, analizar y valorar los rasgos esenciales de esta estrategia internacional universal liderada por la ONU. En el breve espacio del que disponemos, no se tratará más que presentar una síntesis de una estrategia que como tal se basa en dos dimensiones: la institucional operacional a la que nos referiremos en el apartado 3 y la normativa a la que nos referiremos en el apartado 4. Previamente ahondaremos en la historia para entender la razón y trascendencia de los principios operacionales y normativos actuales, en el apartado 2. Finalizaremos con unas breves valoraciones finales sobre este sistema. 


\section{Un poco de historia: la paulatina formación de la estrategia}

Cuando se crea la ONU en 1945, en la Carta de las Naciones Unidas (en adelante también la Carta) no se le atribuye competencia para cooperar en la solución de los problemas en casos de desastres naturales. Ni si quiera para atender a las consecuencias humanitarias de los mismos. La mención en el artículo 1 de la Carta a los "problemas internacionales de carácter humanitario" tenía otra finalidad y justificación. De las actas de la Conferencia de San Francisco se deduce que la voluntad de los Estados fue dar un significado amplio al término humanitario, como una coletilla que cerraba la lista de los tipos de problemas enunciados en dicho artículo (económicos, sociales y culturales) $)^{2}$. Ahora bien, detrás de la letra de la Carta se encontraba la realidad social de una Europa devastada por la guerra, propiciando la creación de los primeros órganos operacionales con fines humanitarios de la Organización.

Los Aliados ya durante la guerra, en 1943, habían creado la primera Organización del Sistema de las Naciones Unidas, con el fin de gestionar el socorro y rehabilitación a los territorios liberados: la Administración de las Naciones Unidas para el Socorro y Rehabilitación (la UNRRA). Inmediatamente creada la ONU, en el primer período de sesiones de la Asamblea General, celebrado en 1946 (en adelante también AG) se estableció el Fondo de las Naciones Unidas para la Infancia- UNICEF (entonces Fondo internacional de socorro a la infancia), a fin de asistir a los niños de los países vencedores de la Segunda Guerra Mundial -discriminación ésta que desaparecerá pronto-. En la práctica, UNICEF hereda la mayoría de fondos de la UNRRA a su liquidación. En 1953 se le da un mandato permanente y sigue funcionando hasta hoy, habiendo ampliado sus funciones operacionales, incluyendo la protección y asistencia a la infancia tras situaciones de desastres naturales y otras similares. También en esos primeros años, la AG establecía el Alto Comisionado de las Naciones Unidas para los Refugiados, en 1950, que reemplazaba a la Organización Internacional de Refugiados (OIR), establecida a su vez en 1946 para atender a los refugiados de la guerra y persecuciones posteriores en la URSS y Europa del Este. ACNUR se crea con mandato temporal y así sigue hoy, renovándose el mandato de la Oficina cada 3 años; hoy presta protección y asistenta a millones de refugiados y desplazados internos (desplazados dentro del propio Estado), en todo el mundo. Sus funciones abarcan también a los denominados en la práctica, refugiados y desplazados ambientales, consecuencias de los desastres naturales.

En 1961, se crea el tercer órgano subsidiario operacional de la ONU con competencia en el sector de los desastres naturales, el Programa Mundial de

\footnotetext{
${ }^{2}$ Para mayor detalle me remito a Torroja Mateu, H., La asistencia humanitaria en la Organización de las Naciones Unidas. Fundamentos y perspectivas actuales, Barcelona, Atelier, 2004, passim.
}

Araucaria. Revista Iberoamericana de Filosofía, Política y Humanidades, año 18, n 36. Segundo semestre de 2016. Pp. 241-263. ISSN 1575-6823 e-ISSN 2340-2199 doi: 10.12795/araucaria.2016.i36.11 
Alimentos (PMA), creado conjuntamente entre la ONU y la Organización para la Agricultura y Alimentación (FAO). Se crea para responder a un problema específico: el del hambre. Su finalidad era establecer una reserva mundial de alimentos desde donde se canalizarían a los países más necesitados por problemas de subdesarrollo o por problemas de emergencia humanitaria en sentido estricto, con el tiempo, incluidas también las crisis provocadas por desastres naturales. Se le dio mandato permanente en 1963 y sigue funcionando activamente en nuestros días. Así, en los primeros quince años de la Organización, se habían creado los órganos operacionales principales de asistencia a las víctimas, incluidas la de desastres naturales, que siguen funcionando hoy.

Por esos años, el proceso de descolonización provocó la aparición de nuevos Estados de reciente independencia, que en general nacían con unas condiciones de desarrollo económico muy inferiores a las de los países industrializados. Se daba a su vez la coincidencia de encontrarse, en su mayoría, en zonas propensas a desastres naturales.

Por ello, el Secretario General empieza a recibir solicitudes de ayuda por algunos de estos países a mediados de los años sesenta. Este momento es destacable porque el problema de los desastres naturales adquiere una entidad propia y específica en la Organización. Su gestión pasaba a ser un interés común de los Estados, una necesidad funcional que conlleva la atribución de funciones a la Organización por los Estados en este sector, generando los cambios institucionales y normativos que veremos. Indiquemos previamente que por aquel entonces la Federación de Sociedades Nacionales de la Cruz Roja y de la Media Luna Roja, creada en 1919, tenía ya bien consolidada su función en el sector de los desastres naturales. Funcionaba a su vez una organización internacional, constituida con el apoyo de la Sociedad de Naciones, en 1927, dedicada a la investigación y estudio sobre la prevención y preparación ante los desastres naturales: la Unión Internacional de Socorro (UIS), que seguiría funcionando hasta su liquidación en 1968.

Entonces, los Estados decidieron atribuir a la Secretaria funciones y poderes específicos en este ámbito, creando un Coordinador de alto nivel que estaría al mando de la Oficina del Coordinador de las Naciones Unidas para el Socorro en casos de desastres, la UNDRO, creada mediante la Resolución 2816 (XXVI) de la Asamblea General, titulada "Asistencia en casos de desastres naturales y otras situaciones de desastre", de 14 de diciembre de 1971. En su adopción se dio ya entonces una tensión subyacente entre la soberanía de los Estados y la atribución de nuevas competencias a la Organización, que se repetirá en los años noventa y aún hoy se sigue repitiendo bajo otros contextos. Preocupaba que la atribución de competencias en materia de desastres naturales y otras situaciones de desastre pudiese ser entendida como una ampliación en materia de conflictos armados. Temían los países del continente suramericano y asiático 
estar dando carta blanca a la ONU para futuras intervenciones humanitarias. Se insistió en que la UNDRO solo tendría competencia en casos de desastres naturales, incluyéndose accidentes industriales, pero no en conflictos armados. Y además actuaría sobre la base de la petición del país afectado, en principio. La UNDRO funcionó hasta 1991, cuando fue reemplazada por el Departamento de Asuntos Humanitarios. Sus veinte años de funcionamiento de la UNDRO fueron años de mejoras y consolidación de experiencias y principios en las dos funciones básicas que le fueron atribuidas: la cooperación en la prevención y preparación ante desastres naturales por una parte (con un fuerte componente de programas de prestación de asistencia técnica para prevenir y prepararse ante desastres naturales), y la movilización y coordinación de los socorros a las víctimas de tales catástrofes (es decir pedir fondos a los Estados y coordinar a los órganos operacionales preexistentes).

Los desarrollos institucionales y normativos adoptados en 1991 por la AG fueron muy relevantes, como veremos, pero todos ellos se basaron en la experiencia anterior, algo que es importante destacar. Por su parte y como siempre, detrás de estas reformas de principios de los noventa, se encontraban unos hechos sociales y la voluntad de varios Estados de atribuir nuevas funciones a la Organización. En 1989 caía el Muro de Berlín y fueron los posteriores años de incremento de conflictos armados, resurgir del genocidio en Europa y África, continuidad de graves desastres naturales... En consecuencia, se incrementaron las actividades humanitarias y reivindicaciones públicas de lo humanitario. Francia fue uno de los Estados pioneros en esta cierta moda del humanitarismo. El movimiento de los french doctors ${ }^{3}$ aboga por reconocer no solo un derecho de injerencia humanitaria - que va a llevarles incluso a actuar contra la voluntad de los Estados, entrando en su jurisdicción sin visados ni autorización- sino en reclamar el reconocimiento de un deber de injerencia ${ }^{4}$, reclamando a su vez una protección jurídica internacional para estos sui generis actores humanitarios, que no respetan alguno de los principios del humanitarismo moderno -en especial el de neutralidad-. Se pedía reconocer internacionalmente, al menos, el derecho de asistir a las víctimas y un derecho de éstas a recibir la asistencia. Francia hará suya esta idea y en 1989, su delegación ante la AG patrocina un proyecto de resolución que dará lugar a la

\footnotetext{
3 Su origen está en la iniciativa de varios médicos franceses, en su mayoría de la generación de mayo del 68, muy altruistas y que durante el conflicto de Biafra (intento de independencia de esta provincia de Nigeria, en el momento de su descolonización, en los años 67-70), siendo trabajadores de la Cruz Roja francesa, deciden dejar de ser neutrales y denunciar públicamente las violaciones de derechos humanos cometidos por el gobierno nigeriano. Esto es, frente a neutralidad (material e ideológica) prefieren la denuncia pública y por tanto posicionarse ideológicamente por una parte en el conflicto. Actúan así, al margen de la protección jurídica internacional existente en el momento.

${ }^{4}$ Deber de injerencia, título de la publicación de las actas de un congreso celebrado en la Universidad de París-Sud, organizado por el Profesor Mario Bettati y el práctico Bernard Kouchner, en 1987. Posteriormente transformado por la expresión derecho de injerencia: Bettati, M., Le Droit d'ingérence: Mutation de l'ordre international, Paris, Odile Jacob, 1996.
} 
Resolución 43/131, titulada "Asistencia humanitaria a las víctimas de desastres naturales y situaciones de emergencia similares". En 1990, la AG adoptaba otra de similar título y contenido, con algunos avances: la Resolución 45/100. Resoluciones recomendatorias, sí, pero que como luego veremos, sí han tenido algunas consecuencias jurídicas. No es momento para detenernos ahora en el contenido y desarrollos de ambas resoluciones. Sí destacaremos que no se lograron los objetivos iniciales de los patrocinadores, fruto de las posiciones de los Estados, celosos de su soberanía. Así, un importante número de Estados, sobretodo latinoamericanos, detrás de las expresiones asistencia humanitaria, derecho de las víctimas a la asistencia, derecho de acceso a las víctimas de los actores, no vieron más que la tradicional figura dela intervención humanitaria, de la que no pocos habían sido víctimas. Nada más lejos del origen de este debate, pero tales Estados presionaron de tal manera que en las resoluciones ni se incluye el derecho humano de las víctimas a la asistencia, ni se incluye la expresión de "conflictos armados" (que quedaría englobada dentro de la expresión situaciones de emergencias similares). Además se insiste en que la responsabilidad en la iniciación, organización, coordinación y prestación de la asistencia es de los Estados afectados y no de instancias exteriores. Así se venía haciendo desde 1971 en la etapa de la UNDRO. Incluso las Resoluciones exigen que la asistencia sea prestada conforme a los principios clásicos del derecho internacional humanitario, incluida la neutralidad, precisamente lo que no querían los movimientos french doctors.

Pero estas dos resoluciones abrieron la puerta y prepararon la base política de los Estados para realizar cambios institucionales y normativos relevantes. Todo ello en un contexto donde lo humanitario se ha puesto de moda en la política de los Estados, entre las organizaciones privadas (se multiplican las ONG humanitarias en estos años), en la propia ONU. En 1991, la AG decidía refundar y fortalecer el sistema de coordinación de la asistencia humanitaria de las Naciones Unidas mediante su Resolución 46/182, Fortalecimiento de la coordinación de la Asistencia humanitaria de emergencia de las Naciones Unidas, aprobada por consenso el 19 de diciembre de 1991. Como apuntamos, la AG establecía que la antigua UNDRO, dedicada sólo a desastres naturales, se transformase en el Departamento de Asuntos Humanitarios, que va a tener funciones de coordinar y liderar el socorro internacional ante todas las catástrofes (esto es, también en los conflictos armados, aunque los Estados no mencionen la expresión que queda escondida bajo "otras emergencias similares" o "situaciones complejas de emergencia")5. Es un avance destacable. Este Departamento será transformado, en 1997, por la Oficina para la Coordinación de Asuntos Humanitarios de la ONU.

${ }^{5}$ Los debates en la Asamblea General dan testimonio de ello. Me remito a Torroja Mateu, H., op. cit., pp. 211-213.

Araucaria. Revista Iberoamericana de Filosofía, Política y Humanidades, año 18, nº 36. Segundo semestre de 2016. Pp. 241-263. ISSN 1575-6823 e-ISSN 2340-2199 doi: 10.12795/araucaria.2016.i36.11 
Así pues, el sistema de la ONU para preservar la seguridad humana en casos de desastres naturales se ha ido formando progresivamente a partir de la respuesta a crisis concretas o a problemas que adquieren relevancia especial en la sociedad internacional. Esto es, detrás de cada nueva institución, de su reforma o de los grandes debates en la AG hay un problema social humanitario que la ONU hace suyo. Problemas sociales que transforman la estructura interna de la Organización y el contenido y alcance de sus funciones normativas. Todo basado en una interpretación extensiva de la noción de "problemas humanitarios" del artículo 1.3 de la Carta de las Naciones Unidas, a través de la práctica de los órganos competentes de su desarrollo, la AG y bajo su dirección, el Consejo Económico y Social (ECOSOC), así como la Secretaría.

\section{Organigrama institucional basado en funciones}

Se pueden distinguir dos grandes ámbitos de la cooperación internacional en torno a los desastres naturales: el de la prevención y preparación ante desastres por una parte, y el de la movilización y coordinación de los socorros, por otra. En base a ello se puede identificar el organigrama institucional de la ONU que cumple funciones en este sector.

\subsection{La función de cooperación para la prevención y preparación ante desastres}

Hay una directriz política fundamental que inspira esta función de cooperación para la prevención y preparación ante desastres que pasamos a exponer. Se basa en la distinción entre el fenómeno natural (los riesgos en sí mismos, como huracanes, maremotos, terremotos, sequías, ciclones, etc.) y el desastre natural en sí (las consecuencias o el impacto de este fenómeno sobre una comunidad dada) ${ }^{6}$. Ello es así porque no siempre que acontece un fenómeno natural tiene lugar un desastre natural; y, si lo tiene, no son siempre equiparables, en abstracto, las magnitudes de su eventual impacto en distintos lugares. Es decir, se considera que el desastre natural no es el fenómeno físico en sí, sino los costes humanos, económicos y sociales provocados por éste. En tal contexto, se constata que ciertos países suelen sufrir con frecuencia desastres naturales, siendo muy a menudo países en vías de desarrollo. Son los conocidos como países propensos o proclives a desastres naturales ${ }^{7}$. El

\footnotetext{
${ }^{6} \mathrm{UN}$, Informe del SG, A/32/64, 1977, p. 15.

7 El SG los define entonces como "los países con un historial de desastres naturales repetidos. Dichos países [son] (...) los países que forman el arco que bordea el Mar de la China Meridional suelen sufrir tifones; la Bahía de Bengala, ciclones; y el Atlántico occidental, la región del Caribe y el Golfo de México, huracanes. Una franja donde con frecuencia ocurren terremotos se extiende desde el Irán 
bajo nivel de desarrollo económico y social de estos países -que se traduce en infraestructuras no preparadas a desastres repentinos, materiales de construcción endebles, edificios colocados en zonas poco propicias, etc.- les convierte además en especialmente vulnerables a sus consecuencias, más desastrosas que en los países más industrializados. Con ello claro se constata una segunda premisa: entre los miembros de la comunidad internacional, existe la capacidad tecnológica y financiera necesaria para reducir las consecuencias de estos desastres en tales países. Un terremoto, por ejemplo, no afecta igual a la población de Japón que a la de India. El primero, a diferencia del segundo, ha aplicado la investigación científica y tecnológica para paliar sus efectos (desde la construcción especial de edificios y carreteras, la elaboración de planes de evacuación, la adopción de una legislación que flexibilice la acción de la administración y otros actores acaecido el desastre, hasta la adopción de mecanismos de alerta temprana sobre futuros seísmos, por ejemplo). Es decir, hay medios tecnológicos y científicos suficientes para reducir los efectos de los desastres y éstos se han de compartir entre todas las naciones. Ésta es la posición técnica de la ONU, basada en los estudios ya iniciados por la UIS y seguidos por la UNDRO. Posición que afirman y hacen suya los Estados que deciden proclamar el decenio de 1990-2000 como Decenio Internacional para la Reducción de los desastres naturales y desde entonces hasta nuestros días sigue siendo una estrategia en la ONU.

Concretando más, cabe indicar que las actividades de prevención se "refieren a los aspectos científicos y de ingeniería de la reducción de los casos de desastres y la legislación conexa". Las segundas, las actividades de preparación (o planificación) incluyen "la preparación de planes para situaciones de alerta y evacuación ante un peligro inminente, así como el rescate y el suministro de alimentos y vivienda a las víctimas una vez ocurrido el desastre" $"$. Estas dos finalidades de la cooperación son atribuidas a diferentes órganos que inciden en su realización a través de diferentes funciones respectivamente: la de armonización de políticas y comportamientos de los Estados a través de estudios, campañas y conferencias; y la de la acción operacional institucionalizada, prestando asistencia técnica. Veamos qué órganos son y qué hacen.

hasta Yugoslavia, pasando por Turquía y Grecia; y la otra, a lo largo de la costa occidental de América. Por último hay una franja a lo largo del bloque septentrional del África donde suelen producirse desastres" (UN, Informe del SG, E/4994, 1971, p. 11, párr. 18).

${ }^{8}$ Por ejemplo, esto incluye "la estimación de la frecuencia y magnitud de los futuros peligros, la delimitación de las zonas de alto riesgo, la determinación de las restricciones que habría que imponer en la utilización de la tierra y la urbanización en esas zonas y el fortalecimiento de estructuras para que resistan a los mayores peligros que sea razonable prever" (UN, Informe del SG, A/45/271-E/1990/78, 1990, p. 14, párr. 34).

${ }^{9}$ Ibidem. Hay aspectos conexos a esta planificación que comprenden el establecimiento de criterios para adoptar la decisión de aplicar los diversos elementos del plan, la capacitación de funcionarios locales y la educación de la población en las zonas de alto riesgo (ibid., p. 14, párr.34). 
3.1.1. UNISDR (Secretaría): la promoción de la prevención y preparación ante desastres

En 1999, la AG decidió establecer la Estrategia internacional para la reducción de Desastres, estableciendo el UNISDR en la Secretaría como entidad competente de su realización. Se considera hoy el órgano central del sistema para asegurar la coordinación y sinergias de todas las actividades de reducción de los riesgos de los desastres, así como de las actividades en el ámbito socioeconómico y humanitario ${ }^{10}$. Era una manera de dar continuidad a los avances logrados por el Decenio Internacional para la Reducción de los desastres naturales (1999-2000), establecido anteriormente por la $\mathrm{AG}^{11}$. La estrategia se basa en la cultura de la prevención que se ha ido reforzando a través de diversas conferencias celebradas posteriormente. Así, la Conferencia internacional de Kobe sobre la prevención de catástrofes naturales (18 a 22 de enero de 2005), donde se adoptó la Declaración Hyogo y Plan de Acción 20052015. Y posteriormente reemplazado en la tercera conferencia mundial sobre Reducción de los Riesgos de Desastres, celebrada el 18 de marzo de 2015 en Sendai, Japón, donde se adopta la Declaración y Plan de Acción Sendai para la Reducción de los Riesgos de Desastres 2015-2030. Se trata de un acuerdo no vinculante que establece una estrategia con un enfoque de seguridad humana, al estar basado en las personas como el centro, y que sustituye al anterior Plan de Acción de Hyogo. El UNISDIR cumple funciones de promoción de la prevención y preparación ante desastres mediante la realización de campañas, la información, la defensa de los principios de la prevención y la coordinación de programas de información ${ }^{12}$.

3.1.2. PNUD (AG): programas de asistencia técnica para la prevención y preparación ante desastres

Desde la reforma de 1997, los poderes operacionales para el ejercicio de la función de cooperación para la prevención y preparación ante desastres pasan a ejercerse por el $\mathrm{PNUD}^{13}$, en lugar de por la OCAH. Originariamente estás funciones las había desempeñado la UNDRO, que realizaba directamente actividades de asistencia técnica en materia de prevención y preparación a los países que lo soliciten. A finales de su existencia, gran parte de estas actividades de asistencia se financiaba con cargo a un Fondo Fiduciario para las actividades de cooperación técnica de la UNDRO. Dada su estrecha relación con la cooperación internacional al desarrollo, en la práctica el PNUD entra a

${ }^{10}$ UN, AG, Resolución 56/195, de 21 de enero de 2002.

${ }^{11}$ UN, AG, Resolución 44/236, de 22 de diciembre de 1989.

${ }^{12} \mathrm{http}: / /$ www.unisdr.org/

${ }^{13} \mathrm{UN}, \mathrm{AG}$, Resolución 52/12 B, de 9 de enero de 1998. 
desarrollar también actividades de asistencia para la prevención y preparación ante desastres naturales. Para evitar una duplicación de esfuerzos se llegó a crear un grupo mixto PNUD-UNDRO encargado de estudiar las formas de coordinarse entre ellos. Se puso ya entonces de manifiesto que sería más conveniente que las actividades de acción paliativa fuesen desempeñadas por el PNUD únicamente ${ }^{14}$. El fortalecimiento de la función se debe al convencimiento de la Oficina de la eficacia de estas medidas y de su reducido coste en comparación con las pérdidas que producen los desastres naturales.

\subsection{La función de movilización y coordinación de la asistencia humanitaria: la OCAH (Secretaría)}

Cuando Kofi Annan asume el puesto de Secretario General una de sus primeras iniciativas es plantear ante la Asamblea General una serie de medidas dirigidas a "reconfigurar profundamente la Organización a fin de que cumpla mejor las tareas que la comunidad internacional le encomiende"; éstas se recogen en su informe titulado "Renovación de las Naciones Unidas: un programa de reforma" ${ }^{15}$. En líneas generales, una primera lectura posible de todas las reformas de Kofi Annan es el tener la finalidad de elevar el nivel y reforzar la esfera de los asuntos humanitarios en la Organización. Por asuntos humanitarios, el Secretario General entiende esencialmente la "coordinación de la acción humanitaria"(actividades de la Secretaría), la "prestación de servicios humanitarios" (actividades del PMA, UNICEF, OMS, y otros) y la "protección y asistencia a los refugiados"(actividades del ACNUR, de la OOPS). Con respecto a las modificaciones institucionales, éstas afectaron tanto a los órganos gubernamentales como a los integrados. En el plano gubernamental, Kofi Annan recomienda reforzar el debate sobre estos temas, asignando al Consejo Económico y Social (ECOSOC) una serie de sesiones sobre asuntos humanitarios. En el marco de la Secretaría, los Asuntos Humanitarios se refuerzan en dos direcciones. Una, mediante la creación del Comité Ejecutivo sobre Asuntos Humanitarios (CEAH o ECHA), uno de los cuatro Comités de este tipo por él ideados; esta propuesta se adoptó sin necesidad de aprobación por la Asamblea General. El CEAH está presidido por el Coordinador de Asuntos Humanitarios y en él participan los Departamentos de la Secretaría y representantes de los principales órganos operacionales humanitarios de la Organización ${ }^{16}$.

${ }^{14}$ Vid. UN, Informe del SG, A/43/731, 1988.

${ }^{15}$ UN, Informe del SG, A/51/950, 1997, p. 2.

${ }_{16}$ Se reúne mensualmente en Nueva York y su mayor ventaja es servir de canal de lo que sería una "coordinación lateral" entre los distintos Departamentos de la Secretaría implicados en cuestiones humanitarias en situaciones complejas de emergencia (como el Departamento de Asuntos Políticos y el Departamento de Operaciones de Mantenimiento de la Paz). Para lograr mayor cohesión entre el CEAH y el Comité Permanente entre Organismos (CPO), la misma sección técnica de la OCHA asiste a ambos. 
La otra dirección de los cambios consistió en la modificación del Departamento de Asuntos Humanitarios (DAH) ${ }^{17}$, que fue reemplazado por una nueva Oficina de la Secretaría que vería reforzadas sus funciones de coordinación de los asuntos humanitarios y dejaría de tener poderes operacionales de asistencia. A su vez, se reduciría su personal y el Coordinador de Socorro de Emergencia pasaría a denominarse Coordinador de la Asistencia Humanitaria de las Naciones Unidas. No se consideraban adecuadas ciertas actividades operacionales de asistencia que se veían como causa del detrimento de la función principal del Coordinador. Así, el Secretario General propone retirar del mandato de la nueva Oficina las funciones relacionadas con las operaciones de prevención y preparación para mitigar los efectos de los desastres naturales, para traspasarlas al Programa de las Naciones Unidas para el Desarrollo (PNUD), como ya se indicó. Decide también retirarle las funciones relativas a la remoción de minas y cuestiones conexas, así como la gestión del Fondo Fiduciario Voluntario de la Asistencia para la Remoción de Minas, para ser transferidas al Departamento de Operaciones de Mantenimiento de la Paz; eran funciones que tenía por atribución directa del anterior Secretario General, habiendo sido posteriormente aceptado por la Asamblea General (Resolución 49/215, 1994, preámbulo y punto 2 parte dispositiva). Y por último, decide retirar la gestión del Programa para Iraq "Petróleo por alimentos" establecido en virtud de la Resolución 986 (1995) del Consejo de Seguridad, que pasaría a estar a cargo de una dependencia especial de la Secretaria. Todas las propuestas de reforma son adoptadas.

Las funciones de la Oficina ampliamente enunciadas en la Resolución 46/182, se concretan en tres: 1) la formulación y coordinación de políticas; 2) la promoción de las cuestiones humanitarias; y 3) la coordinación de la respuesta humanitaria en casos de emergencia. Para el ejercicio de estas funciones se le dotaron de diversos instrumentos de coordinación en la sede y en el terreno ${ }^{18}$. En la práctica, contrasta el constante deseo de los Estados por reforzar la OCHA, con la falta de asignación de fondos a la misma, llevándole a graves problemas de financiación. No es un rasgo nuevo en el sector humanitario de la ONU.

${ }^{17}$ Establecido por la Asamblea General por la Resolución 46/182 relativa al "Fortalecimiento de la coordinación de la asistencia humanitaria de emergencia del sistema de las Naciones Unidas", de 19 de diciembre de 1991.

18 Como los siguientes: Los llamamientos unificados interinstitucionales para coordinar la financiación. El Fondo Central para la acción en casos de emergencias que es un mecanismo de financiación de reserva, común a todo el sistema. El fondo se financia con contribuciones voluntarias. También se establecen fondos humanitarios comunes que iniciaron algunos donantes para ayudar a los coordinadores humanitarios que están sobre el terreno un mayor grado de acceso y autoridad sobre unos fondos predecibles y flexibles que pudieran desembolsarse con arreglo a las necesidades y las prioridades. Se establecen Coordinadores Humanitarios y se refuerza la formación en asuntos humanitarios a los Representantes Residentes. Otros mecanismos de coordinación sobre el terreno consisten en la designación de un órgano operacional como organismo rector (lead agency) u órgano principal (focal point).

Araucaria. Revista Iberoamericana de Filosofía, Política y Humanidades, año 18, no 36. Segundo semestre de 2016. Pp. 241-263. ISSN 1575-6823 e-ISSN 2340-2199 doi: 10.12795/araucaria.2016.i36.11 
Ello fue constante en la etapa de la UNDRO. Siendo parte de la Secretaría y siguiendo la trayectoria de la UNDRO, los gastos administrativos se financian con cargo al presupuesto ordinario. Si bien se ha señalado que en la práctica ha habido una importante parte del personal que se ha financiado con cargo a fondos extrapresupuestarios. Para los gastos operacionales, el Departamento ha contado con la gestión de diversos Fondos Fiduciarios financiados con contribuciones voluntarias.

LAOCHA presta servicios técnicos al Comité Permanente entre Organismos (CPO) que cumple una función de coordinación entre los órganos de gestión humanitarios. Es un foro, presidido por el Coordinador, donde se reúnen los Directores Ejecutivos y los Directores Generales (u otros) de los órganos y organismos operacionales competentes en la esfera humanitaria o que puedan tener especial interés del Sistema de las Naciones Unidas (UNICEF, ACNUR, PNUMA, PNUD, OMS, FAO); en la Resolución también se invita a tener una representación permanente al CICR, la FICR y a la Organización Internacional para las Migraciones (OIM), pudiéndose invitar también a distintas $\mathrm{ONG}^{19}$. Una iniciativa del CPO fue establecer un enfoque por grupos temáticos (clusters), que tiene por finalidad proporcionar una respuesta humanitaria más oportuna, predecible y eficaz a las poblaciones necesitadas. Buscan identificar el portavoz único de la comunidad internacional ante el gobierno afectado. Se trata de un sistema de once grupos de trabajo, dirigido cada uno por una organización competente, en los que participan agentes humanitarios de dentro y fuera de las Naciones Unidas que desempeñan su labor en esferas que carecían de una dirección y una capacidad predecibles ante las emergencias humanitarias o que requerían una asociación más estrecha con otros agentes humanitarios ${ }^{20}$.

\section{La función normativa}

La ONU cumple a su vez una función normativa en el ámbito de los desastres naturales, con la finalidad de coordinar las politicas y comportamientos de los Estados. El órgano principal competente es la AG y podemos identificar

19 Se ha invitado a diferentes agrupaciones de ONG. Y posteriormente se ha invitado a participar también al Alto Comisionado de las Naciones Unidas para los Derechos Humanos (ACNUDH), al Banco Mundial y al Representante del Secretario General sobre Desplazados Internos, entre otros. Para la composición actual véase: (http://www.humanitarianinfo.org/iasc/membership.asp).

20 Agricultura (FAO); Coordinación y gestión de campamentos (ACNUR y OIM. Recuperación temprana (PNUD). Educación en las emergencias (UNICEF y Save the Children del Reino Unido). Alojamiento de emergencia (ACNUR y FICR). Telecomunicaciones de emergencia (OCHA/ UNICEF/PMA). Salud (Organización Mundial de la Salud, OMS). Logística (PMA). Nutrición (UNICEF). Protección de personas desplazadas por los conflictos (ACNUR). Protección de personas desplazadas por motivos que no son conflictos (UNICEF/ACNUDH/ACNUR). Agua/saneamiento/ higiene (UNICEF). 
dos formas de contribución: una, directamente mediante la adopción de Resoluciones; otra, mediante los trabajos de codificación y desarrollo progresivo, principalmente a través de su Comisión de Derecho Internacional.

Antes de pasar a indicar los resultados más relevantes, conviene situar estas normas en el contexto de lo que otrora se llegó a denominar como el derecho internacional de los desastres y hoy continúa siendo de actualidad ${ }^{21}$. Así por ejemplo, el profesor LEBEN se refiere a la posibilidad de la configuración de un Derecho de las Catástrofes (Droit des Catastrophes). Pero se trata de una configuración en proceso, cuya consolidación como rama específica encuentra sus dificultades por hallarse su regulación dispersa en distintos sectores normativos sí consolidados (derecho internacional del medio ambiente, derecho internacional humanitario, derecho de la responsabilidad internacional...).

\subsection{Principios jurídicos adoptados por la Asamblea General}

La Resolución 46/182 de la AG ya citada vino a dar continuidad a los principios rectores de la prestación de asistencia humanitaria introducidos a través de las Resoluciones 43/131 y 45/100, así como a través de las distintas resoluciones adoptadas en la etapa de la UNDRO, mencionada en su preámbulo. En su Anexo se establecen con claridad bajo el título de "principios rectores", expresión que refleja la intención de dar un contenido normativo a sus disposiciones. Los principios se refieren a la prestación de asistencia humanitaria tanto en situaciones de desastres naturales como en otras situaciones entre las que se incluyen los conflictos armados. Su alcance jurídico se puede interpretar de la siguiente manera.

\subsubsection{Obligaciones y derechos del Estado afectado}

La primera cuestión es la siguiente. ¿Está el Estado donde ha ocurrido la catástrofe obligado a prestar asistencia a las víctimas? La respuesta es clara: sí. Todas las resoluciones relevantes de la Asamblea General desde los años setenta (la etapa de la internacionalización de los desastres naturales) han afirmado "la responsabilidad principal que tiene el Estado de ocuparse de las víctimas de desastres naturales y de otras urgencias. Tiene la responsabilidad de iniciar, organizar, coordinar y prestarles asistencia humanitaria en su territorio",22. En el debate histórico, los Estados incluyeron esta referencia a su responsabilidad para defenderse de las intervenciones exteriores; pero, indirectamente, ello ha

\footnotetext{
${ }^{21}$ Caron, D. D. et al (Edit.), The International Law of Disaster Relief, Cambridge, Cambridge UP, 2014.

22 UN, AG, Res. 46/182, 1991.
}

Araucaria. Revista Iberoamericana de Filosofía, Política y Humanidades, año 18, $\mathrm{n}^{\circ} 36$. Segundo semestre de 2016. Pp. 241-263. ISSN 1575-6823 e-ISSN 2340-2199 doi: 10.12795/araucaria.2016.i36.11 
dado lugar a un efecto externo, llevando a la formulación de tal obligación en este ámbito. De hecho, esta obligación está reconocida en el marco del Derecho Internacional Humanitario: las Convenciones de Ginebra y los Protocolos Adicionales la establecen para las partes implicadas en los conflictos internacionales y en los internos (artículo 3 común). Pero esto sólo puede afirmarse con relación a los conflictos armados. En el resto de emergencias humanitarias no existe una regulación internacional convencional tan precisa. En estos casos se puede considerar que las resoluciones de la Asamblea General al respecto son declarativas de un principio general del Derecho internacional: la obligación general de respeto del derecho a la vida, uno de los derechos humanos fundamentales y el núcleo duro del Principio de humanidad, en su dimensión activa de prestar asistencia humanitaria.

Por el consenso con que se aprobó la Resolución 46/182 y tal como está redactado el punto 4 de los Principios rectores de la misma es posible considerar su alcance jurídico vinculante. Han de recordarse los antecedentes y fundamentos históricos de esta afirmación en la ONU: los Estados la vienen formulando en la Asamblea General desde al menos 1971, ya fuera de forma expresa o implícita; entonces se hacía desde la perspectiva de los desastres naturales y "otras situaciones de desastre". No es por tanto una afirmación nueva. Podría decirse que el conjunto de todas las resoluciones de la Asamblea General que reconocen esta responsabilidad principal (identificando aquí responsabilidad con obligación) son el reflejo de una costumbre internacional. En mi opinión esta afirmación por la Asamblea General no sería sino una concreción del Principio de humanidad, considerado como un Principio General del Derecho Internacional que enuncia una norma de ius cogens y una obligación erga omnes. La Asamblea General vendría con el punto 4 de la Resolución 46/182 a declarar concretando un Principio General del Derecho, el Principio de Humanidad en su vertiente de la prestación de asistencia humanitaria extendido a tiempos de paz. Principio que hoy por hoy impone obligaciones al Estado con respecto a las personas bajo su jurisdicción.

Sin embargo, ante la norma jurídica el problema lo pone de manifiesto la realidad social. ¿Qué pasa cuando un Estado no puede o no quiere ocuparse de las víctimas bajo su jurisdicción? ¿Está obligado a aceptar los socorros que le proponen los terceros Estados, la ONU o las ONG? Las resoluciones de la Asamblea General no lo afirman expresamente. En este punto están redactadas en términos de recomendación pero no de obligación. Los Estados no han querido reconocer formalmente tal obligación ante la Asamblea General. En el ámbito de los conflictos armados, los Convenios de Ginebra sí contemplan tal obligación, si bien en algunos casos puede estar exceptuada si las necesidades militares lo requieren. Es destacable al respecto que el Estatuto de Roma, en el artículo 8, 2, b) XXV, contemple como un crimen de guerra "hacer padecer 
intencionalmente hambre a la población civil como método de hacer la guerra, privándola de los objetos indispensables para su supervivencia, incluido el hecho de obstaculizar intencionalmente los suministros de socorro de conformidad con los Convenios de Ginebra".

En los otros ámbitos del Derecho internacional no está tan clara la existencia de tal obligación relativa a la aceptación de la ayuda. Alguna doctrina afirma la posibilidad de su formulación jurídica por analogía con el Derecho internacional humanitario y por la obligación de cooperar con la ONU enunciada en el artículo 56 de la Carta de las Naciones Unidas. En mi opinión, no es tanto al derecho a quien corresponde aportar una solución sino a las negociaciones diplomáticas del Coordinador de Asuntos Humanitarios, o del Secretario General mismo o de su Representante especial sobre el terreno. Pero, si el Estado sigue rechazando recibir una asistencia humanitaria, ¿se puede imponer, incluso mediante la fuerza armada? Surge aquí el problema de la denominada injerencia humanitaria y de la figura de la intervención humanitaria, hoy escondida o reformulada bajo la expresión "responsabilidad de proteger".

\subsubsection{Obligaciones y derechos de terceros Estados}

Otra cuestión es si los terceros Estados están obligados a prestar asistencia humanitaria. En este ámbito las resoluciones más destacadas de la Asamblea General se redactan también en términos de recomendación y no de obligación. Lo cierto es que ni en el Derecho internacional humanitario, el más detallado en la materia de los socorros o asistencia humanitaria, se recoge una tal obligación de los terceros Estados de prestar asistencia (si bien, sí hay algunas obligaciones para los terceros Estados de acordar el libre paso de los socorros, aunque no de forma absoluta). En algunos casos, puede haber convenios bilaterales de asistencia mutua o Convenios regionales, como en el marco de la Organización de Estados Americanos (OEA) ${ }^{23}$. Pero no hay convenio universal alguno que lo establezca. Hay que mencionar que existe una regulación dispersa y fraccionada para agilizar la prestación de socorros relativa a exenciones aduaneras, facilitación del transporte internacional aéreo y terrestre, utilización de recursos de telecomunicación... Pero es un derecho sectorial y particular, esto es, que sólo obliga a las partes en los convenios internacionales concernientes; su objeto y fin es el de facilitar la prestación de asistencia humanitaria, pero no el de obligar a prestarla en sentido estricto y con alcance general.

${ }^{23}$ Convención interamericana para facilitar la asistencia en casos de desastre, de 7de junio de 1991, adoptada en el marco de la Organización de Estados Americanos, en vigor desde el 16 de octubre de 1996; texto en OEA, AG/Res. 1101 (XXX-0/91).

Araucaria. Revista Iberoamericana de Filosofía, Política y Humanidades, año 18, n 36. Segundo semestre de 2016. Pp. 241-263. ISSN 1575-6823 e-ISSN 2340-2199 doi: 10.12795/araucaria.2016.i36.11 
Desde un punto de vista doctrinal se podría considerar que los Estados más poderosos deberían estar obligados a socorrer a las poblaciones afectadas de los Estados menos favorecidos. Así lo afirma alguna doctrina y de la interpretación propuesta de la Carta de las Naciones Unidas, podría derivarse la existencia de una obligación en tal sentido. Pero, la práctica de los Estados va muy en sentido contrario, por lo que no estoy segura de que exista tal obligación internacional. Antes bien parece que los Estados prestan la asistencia humanitaria porque quieren prestarla en determinadas ocasiones, pero no porque cumplan una obligación internacional. Ejemplo indirecto de ello es que las contribuciones para financiar los programas de socorro, son voluntarias.

\subsubsection{Obligaciones de la comunidad internacional a través de la ONU}

Cabe por último preguntarse si la ONU en tanto que actor autónomo de las relaciones internacionales (y quien dice ONU ahora está pensando en UNICEF, ACNUR, PMA, el Coordinador de Asuntos Humanitarios) está obligada a prestar sus servicios humanitarios. En algún momento el Secretario General Kofi Annan ha hablado de un "deber moral" de la ONU de prestar tal asistencia. Pero no puede hacerse esta interpretación. En la teoría general de las Organizaciones internacionales se afirma que la atribución de competencias por los Estados a la Organización es precisamente lo que justifica su existencia. A diferencia de los Estados, que no han de justificar su propia existencia, las Organizaciones internacionales sí; esto es, lo que las justifica son sus funciones y éstas se han de cumplir; así lo ha señalado la Corte Internacional de Justicia ${ }^{24}$.

Por lo tanto, si la ONU tiene atribuida por los Estados una función de realizar la cooperación internacional en la esfera humanitaria, como hemos visto, ello quiere decir que está obligada a su ejercicio. Lo que implica que al menos está obligada a prepararse y ofrecer sus servicios. No es un derecho solamente, sino un deber. Creo que esto es una idea importante porque cuando la ONU presta asistencia a través de ACNUR, PMA, UNICEF o la OCHA no está haciendo humanitarismo (o caridad o beneficencia o como se quiera llamar...), sino que está cumpliendo con un deber, una función jurídica, lo que es algo muy distinto. Incluso no es necesario que la Organización espere la petición de un país afectado. Durante años, las resoluciones de la Asamblea General insistían en la necesidad de esta petición. Pero en 1991, se logra introducir un matiz en su redacción ("en principio"), que permitiría entender que puede iniciar a ofrecer sus servicios sin esperar a la petición.

Ahora bien, la ONU siempre se va a encontrar con el límite de la soberanía del Estado afectado. Ello es un aspecto general para todas las actividades

${ }^{24}$ Opinión consultiva sobre la reparación de daños sufridos al servicio de las Naciones Unidas, 11 de abril de 1949, CIJ, Recueil, 1949. 
operacionales de las Organizaciones internacionales en el marco de la cooperación: necesitan el consentimiento del Estado receptor para la entrada en su territorio. Ello quiere decir que la iniciativa de la ONU puede verse frustrada si el Estado afectado rechaza dejarle entrar. Pero a su vez, y por otra parte, la ONU sólo puede hacer lo que los Estados le dejan hacer. Y si éstos no le asignan los recursos financieros suficientes, ¿qué puede hacer la ONU? Al respecto, la crisis financiera en el sector humanitario, sufragado en su mayor parte con contribuciones voluntarias, ha sido una constante en la historia de la función de la ONU en este ámbito. A ello añádase una tendencia reciente: en ciertas ocasiones los Estados se salen del marco multilateral onusiano para prestar asistencia humanitaria unilateralmente. Por ejemplo, como todo lo que es humanitario vende bien frente a la opinión pública, algunos Estados politizan lo que es humanitario, buscando ser líderes de la solidaridad.

En cualquier caso, la asistencia humanitaria deberá prestarse siempre respetando los principios de humanidad, neutralidad e imparcialidad, reiterados en las resoluciones de la AG.

\subsection{Codificación y desarrollo progresivo de las normas relativas a la protección y asistencia en casos de desastres naturales}

Hay dos iniciativas de codificación de estas normas. La primera, de los años ochenta, no prosperó. La segunda, contemporánea, está en su segunda fase de elaboración.

4.2.1. El "Proyecto de Convención propuesto para acelerar la prestación de socorro de emergencia" de la UNDRO de 1984

La UNDRO intentó que los Estados se comprometieran convencionalmente para agilizar la prestación de la asistencia humanitaria en casos de desastres naturales. Con tal intención, preparó un "Proyecto de Convención propuesto para acelerar la prestación de socorro de emergencia", presentado en $1984^{25}$. $\mathrm{Su}$ ámbito de aplicación se limitaba únicamente a los supuestos de desastres naturales, excluyendo expresamente el ámbito de los conflictos armados. El Proyecto, examinado por un grupo informal de expertos y representantes de Organizaciones Internacionales y de ONG, se presentó al ECOSOC con la intención de que se decidiese si convenía o no su revisión por expertos gubernamentales. Sin embargo, no fructificó la idea y la iniciativa quedó frustrada. Su contenido refleja un gran detallismo, tratando los problemas

\footnotetext{
${ }^{25}$ Publicado en UN, Informe del Coordinador del Socorro: A/39/267/Add.2-E/1984/96/Add.2, 1984. 
fundamentales de la prestación de asistencia humanitaria ${ }^{26}$. Sus disposiciones se basaban en el respeto de la soberanía del Estado, no pudiendo ser de otra manera.

4.2.2. El Proyecto de artículos en primera lectura de la CDI sobre "Protección de las personas en caso de desastre" de 2014

Este tema fue iniciado por la Comisión de Derecho internacional (CDI) en 2008. En 2016, se aprueba un proyecto de 18 artículos con su comentario, que se remite a la Asamblea General para su consideración, recomendando su adopción como tratado internacional ${ }^{27}$.

El objeto del proyecto de artículos es codificar y desarrollar progresivamente las normas internacionales relativas a la protección de las personas en casos de desastres (art. 1), a fin de "facilitar una respuesta adecuada y eficaz a los desastres, que atienda las necesidades esenciales de las personas afectadas respetando plenamente sus derechos" (art. 2).

El ámbito de aplicación material del proyecto es el de los "desastres", optando por una definición abstracta y lo más amplia posible de éstos, sin dejar por ello de elevar el umbral de los mismos a los efectos de limitar su internacionalización. Se evita seguir la distinción entre el fenómeno natural y el desastre, a mi entender un error de la CDI. Voluntariamente se decide omitir cualquier mención expresa de la tipología de desastres (desastres naturales como huracanes, terremotos, etc., o accidentes industriales...) y no queda claramente resuelto, desde mi perspectiva, la cuestión de si las situaciones de conflicto armado -supuesto clásico y originario de la regulación internacional de la protección y asistencia a sus víctimas- entran detrás de la noción de "desastres". A efectos prácticos, el proyecto sí tiene por finalidad abarcar también la aplicación a las situaciones de conflicto armado donde pueda tener lugar una situación de "desastre". Claramente lo afirma la CDI en su comentario al artículo $18^{28}$.

${ }^{26}$ Vid. el índice del proyecto: Parte I.- Disposiciones Generales: Art. 1: Definiciones; art. 2: Objetivo de la Convención; art. 3: Principios; art. 4: Alcance de la Convención. Parte II.Asistencia: art. 5: Asistencia; art. 6: Intercambio de información; art. 7: Protección y facilidades; art. 8: Comunicaciones; art. 9: Notificación; art. 10: Carácter cualitativo de la asistencia; art. 11: Embalaje, etiquetas y marcas; art. 12: Identificación; art. 13: Exportación; art. 14: Importación; art. 15: Disposiciones financieras; art. 16: Contabilidad; art. 17: Irregularidades; art. 18: Terminación de la asistencia. Parte III.- Transporte: art. 19: Facilidades; art. 20: Tránsito; art. 21: Transporte aéreo. Parte IV.- Responsabilidad: art. 22: Responsabilidad; Parte V.- Disposiciones Finales (art. 23 a 33 ) Apéndice: Impreso modelo para documentación de importación/exportación (Ibid.).

27 "Draft articles on the protection of persons in the event of disasters, with commentaries", 2016, $\mathrm{A} / 71 / 10$.

${ }^{28}$ Así, se afirma que "In this manner the present draft articles will contribute to filling possible legal gaps in the protection of persons affected by disasters occurring during an armed conflict" (CDI, Informe A/71/10, doc. cit., p. 136). 
En relación al contenido jurídico - derechos y obligaciones para los sujetos internacionales-, podemos destacar lo siguiente, en comparación con los principios adoptados por la Asamblea General en 1991, y la interpretación que he realizado de los mismos.

El proyecto no hace mención expresa del "derecho a la asistencia", ni del "derecho a los alimentos y bienes de subsistencia", ni expresión alguna de un "derecho humano a la asistencia humanitaria". Sí fundamenta todo el articulado en la dignidad inherente a la persona humana (art. 4) y en el derecho de las personas afectadas por los desastres al respeto de los derechos humanos (art. 5).

El proyecto identifica las siguientes obligaciones y derechos del Estado afectado. Muy acertadamente, a mi entender, establece el "deber de reducir el riesgo de desastres" (art. 9). No establecido como tal deber en los Principios rectores de la AG antes estudiados, era sin embargo una directriz que venía destacándose desde 1971, desde la creación de la UNDRO. Entiendo que sería una codificación de un principio general o una costumbre preexistentes.

En relación a la prestación de asistencia humanitaria -utiliza el proyecto el término clásico de prestación de socorro-, destaca el reconocimiento del "deber de protección y asistencia a las personas en su territorio" (art. 10.1), aclarando el comentario de la CDI que debe leerse en su acepción de "jurisdicción". También se trata de codificación de principio general o costumbre internacional, ya reconocido por los Principios rectores de la AG. Acertadamente, se incluye una concepción funcional de la soberanía, expresamente mencionada en este párrafo primero del proyecto de artículo 10. Establece el "deber del Estado afectado de buscar asistencia externa" (art. 11), algo novedoso, un avance de desarrollo progresivo, sobre lo que nada establecían los Principios rectores de la Resolución de la AG 46/182 de 1991; si bien, queda salvaguardada la soberanía del Estado, ya que la obligación solo es "en la medida en que un desastre supere manifiestamente su capacidad nacional de respuesta". Y además la obligación es de "buscar", que sería, entiendo, "pedir", "solicitar", o formular un "llamamiento internacional". Se complementa esta obligación con la prohibición de denegación arbitraria de la asistencia (art. 13.2) y la obligación de comunicar, cuando sea posible, la decisión sobre el ofrecimiento de asistencia externo (art. 13.3). Ambos aspectos serían un desarrollo progresivo en el ámbito de los desastres naturales claramente, y con algunos matices en el marco del Derecho internacional humanitario.

Se obliga al Estado afectado a "facilitar" la entrada y la prestación de la asistencia, concretándose los ámbitos de esta facilitación en personal, equipo y bienes de socorro (art. 15). Se concreta a su vez la obligación de protección de tal personal y bienes (art. 16). Es una concreción del deber general de protección bien establecido en el Derecho internacional público. Es a mi 
entender, codificación de un principio general o una costumbre, fundamentado en el principio general del Derecho internacional relativo al Deber general de protección que tiene el Estado con relación a los bienes y personas que se encuentran bajo su jurisdicción.

Se establece también el deber de consulta y dialogo en relación a la terminación de la asistencia externa entre el Estado afectado y los Estados y otros actores que prestan la asistencia (art. 17).

En cuanto a los derechos del Estado afectado, se reconoce su derecho a iniciar, dirigir y liderar la prestación de socorro y asistencia (art. 10.2). Sería una codificación de un principio general, siguiendo la línea de los Principios rectores de la AG. A su vez, el Estado tiene el derecho de decidir si acepta o no la asistencia externa; el consentimiento del Estado está pues protegido (art. 13.1), con la única salvedad de la prohibición de denegación arbitraria ya citada. Es por supuesto, codificación de una norma general ya reconocida por la AG. Se le reconoce al Estado afectado el derecho de establecer condiciones a la prestación de la asistencia externa, con la única limitación de que éstas "deberán tener en cuenta las necesidades identificadas de las personas afectadas por el desastre y la calidad de la asistencia" (art. 14).

Por otra parte, el proyecto establece los rasgos obligatorios de la asistencia, los "principios humanitarios" clásicos (art. 6), humanidad, neutralidad e imparcialidad, que de forma indeterminada, deben respetar todos aquellos que presten asistencia.

\section{Consideraciones finales}

De todo lo visto hasta ahora se pueden destacar las siguientes consideraciones. En el plano universal, la acción de la ONU conforma un valioso acervo de órganos y principios dirigidos a mitigar las consecuencias de los desastres naturales, todos ellos basados en el enfoque de la seguridad humana, esto es situando al ser humano en el centro. La estrategia de la ONU se basa en dos grandes funciones: la prevención y preparación ante desastres naturales y la movilización y coordinación de la asistencia humanitaria a las víctimas, incluida la de los propios órganos operacionales de la ONU. Del desarrollo de ambas funciones se encargan diversos órganos operacionales y con respecto a ambas finalidades se han adoptado directrices políticas e incluso normativas.

La función de prevención y preparación ante desastres no solo se lleva a cabo a través de su promoción mediante campañas internacionales y conferencias (a través de UNISDIR), sino también mediante la prestación de asistencia técnica en este sector en el marco de la cooperación internacional 
al desarrollo (a través del PNUD). El sistema actual es la evolución lógica de unos principios y formas de actuación iniciados a finales de los años sesenta, tras la descolonización y el aumento de Estados propensos a desastre en la sociedad internacional. Por otra parte, en el plano normativo, el actual proyecto de artículos de la CDI sobre "Protección de las personas en caso de desastres", tiene el valor de haber incluido como una obligación del Estado afectado desempeñar esta función de prevención y preparación ante desastres.

En la otra vertiente funcional, la de la acción humanitaria operacional, el sistema establecido en la ONU no es exclusivo para los casos de desastres naturales. Antes bien, la tendencia ha sido la de integrar los diversos supuestos de hecho (conflictos armados incluidos) dentro del marco de las competencias de los órganos operacionales que antes únicamente se centraban en casos de desastres naturales. Así, la OCHA actualmente dirige sus funciones de movilización y coordinación de los socorros hacia todos los supuestos de hecho. En este ámbito, cabe destacar la función normativa de la Asamblea General, que en 1991 proclama unos principios rectores de la asistencia humanitaria, aplicables a todos los supuestos y con una clara vocación normativa general. Si bien algunos de ellos pueden considerarse como declarativos de principios generales del derecho o costumbres internacionales, es cierto que no dejan de ser derecho internacional general. Por ello, la CDI ha realizado satisfactoriamente una labor de codificación y desarrollo progresivo de los mismos a través del proyecto supra citado. 


\section{Referencias bibliográficas:}

AAVV, III Jornadas de Derecho Internacional Humanitario: El Derecho de Injerencia por Razones Humanitarias, Sevilla, Cruz Roja Española, Universidad de Sevilla y la Asociación para las Naciones Unidas en Sevilla, 1995.

AAVV, La Asistencia Humanitaria en Derecho Internacional Contemporáneo, Sevilla, Secretariado de Publicaciones de la Universidad de Sevilla, 1997.

Bettati, M. y B. Kouchner (Edit.), Le devoir d'ingérence - Peut-on les laisser mourir?, París, Denoël, 1987.

Bettati, M., Le Droit d'ingérence: Mutation de l'ordre international, Paris, Odile Jacob, 1996.

Blondel, J-L., L'assistance aux personnes protégées, "Revista Internacional de la Cruz Roja", No. 767, septiembre-octubre, 1987, pp. 471-489.

Caron, D. D. et al (Edit.), The International Law of Disaster Relief, Cambridge, Cambridge UP, 2014.

Casanovas i la Rosa, O., De l'ajuda humanitària al dret d'ingerència humanitària”, Lliçó inaugural del Curs acadèmic 1992-93, Barcelona, Publicacions de la Universitat Pompeu Fabra, 1994.

Destexhe, A., L'humanitaire impossible ou deux siècles d'ambigü̈té, Paris, Armand Colin, 1993.

Fischer, H. y Oraá, J., Derecho Internacional y Ayuda Humanitaria, Bilbao, Universidad de Deusto, 2000.

Giles Carnero, R. M., De la asistencia a la injerencia humanitaria: la práctica reciente del Consejo de Seguridad, Sevilla, Universidad de Huelva-Cruz Roja Española en Huelva, 1997.

Green, S., International Disaster Relief, Toward a Responsive System, New York, Mc Graw-Hill Book Company, 1977.

Institut de Droit Internacional, Resolución Santiago de Compostela III. La protection des droits de l'homme et le principe de non-intervention dans les affaires intérieures des Etats, "Annuaire de l'Institut de Droit International", Vol. 63-II, 1990, pp. 338-345.

Institut International de Droit Humanitaire de San Remo, Principes directeurs concernant le droit à l'assistance humanitaire, "Revista Internacional de la Cruz Roja”, No. 804, noviembre-diciembre, 1993, pp. 548-554.

Kent, R. C., Anatomy of Disaster Relief: The International Network in Action, 1987, Pinter Publishers Limited, 1987.

Langeais, G., Les Nations Unies face aux catastrophes naturelles: Étude du Bureau du Coordinateur des Nations Unies pour les Secours en cas de catastrophe (UNDRO), París, LGDJ, 1977. 
Leben, C., Bilan de recherches de la section de langue française du Centre d'étude et de recherche de l'Académie, Académie de Droit International de La Haye. Centre d'étude et de recherche de Droit International et de Relations Internationales, 1995: Les aspects internationaux des catastrophes naturelles et industrielles, Dordrecth, Martinus Nijhoff Publishers, 1996.

Macalister Smith, P., International Guidelines for Humanitarian Assistance Operations, "HumanitäresVölkesrecht", Año 3, Julio-diciembre, 1990, pp.143-146.

Macalister Smith, P., The Right to Humanitarian Assistance in International Law, "Revue de Droit International de Sciences Diplomatiques et Politiques", julio-septiembre, No. 3, 1988, pp. 211-233.

Macalister-Smith, P., International Humanitarian Assistance: Disaster Relief Actions in International Law and Organization, Dordrecht, Martinus Nijhoff Publishers. Dordrecht, 1985.

Paye, O., Sauve qui veut? Le Droit International face aux crises humanitaires, Bruselas, Bruylant, 1996.

Rufin, J-C., Le Piège humanitaire (suivi de Humanitaire et Politique depuis la chute du Mur).París, Pluriel 2a ed., 1993.

Stephens, L. H. y J. G. Stephen (Edit.), Disaster Assistance-Appraisal, Reform and new approaches, New York, New York University Press, 1979.

Stephens, T., The United Nations Disaster Relief Office: The Politics and Administration of International Relief Assistance, Washington, University Press of America, 1978.

Thouvenin, J. M., L'internationalisation des secours en cas de catastrophe naturelle,"RGDIP", 1998/2. pp. 327-363.

Torroja Mateu, H., Cooperación internacional y desastres naturales: la estrategia de la Organización de las Naciones Unidas, "Areas”, No. 23, 2003, pp. 143-157.

Torroja Mateu, H., La asistencia humanitaria en la Organización de las Naciones Unidas. Fundamentos y perspectivas actuales, Barcelona, Atelier, 2004.

Torroja Mateu, H., La función de cooperación internacional en asuntos humanitarios de la ONU, "Revista Jurídica Universidad de Puerto Rico", Vol. 75, No. 3, 2006, pp. 703-720. 
